CME-Herausgeber- und Review-Board:

Hier können Sie CME-Punkte sammeln a) für die Pflichtfortbildung aller Vertragsärzte und b) für freiwillige Fortbildungszertifikate, die viele Landesärztekammern anbieten.

Die Multiple-Choice-Fragen beziehen sich auf den vorangegangenen Fortbildungsbeitrag (Seiten 46-49). DIE ANTWORTEN ERGEBEN SICH Z. T. AUS DEM TEXT, Z. T. BERUHEN SIE AUF MEDIZINISCHEM BASISWISSEN!

Wenn Sie 70\% der Fragen richtig beantworten, erhalten Sie 2, bei 100\% 3 CME-Punkte.

Einsendeschluss: 15. März 2008 (www.cme-punkt.de) bzw. 29. November 2007 (Teilnahme per Brief).
Dr. H. J. K. Barwitz, Prof. Dr. A. Berghaus, Prof. Dr. Dr. h.c. Th. Brandt, Prof. Dr. W. G. Daniel, Prof. Dr W. Eisenmenger, Prof. Dr. K. Friese, Prof. Dr. H.

S. Füessl, Prof. Dr. B. Göke, Prof. Dr. R. Gradinger, Prof. Dr. Dr. h.c. A. Hofstetter, Prof. Dr. H. Holzgreve, Prof. Dr. A. Imdahl, Prof. Dr. K. Krüger, Prof. Dr. H.-J. Möller, Prof. Dr. Dr. h.c. G. Plewig, Prof. Dr. D. Reinhardt, U. Weigeldt.

\title{
Fragen zum Thema „Krankhafte Sorge“
}

\author{
- 1. Welches ist das führende Diagnose- \\ merkmal der GAS nach DSM-IV? \\ A Reizbarkeit \\ B Ruhelosigkeit \\ C Schlafstörungen \\ D Muskelspannung \\ E Pathologische Sorgen
}

\section{-2. Welches Alter ist bei der Erstmanifestation typisch?}

A Jugend- bzw. frühes Erwachsenenalter

B Zwischen 30 und 40 Jahren

C Zwischen 40 und 50 Jahren

D Zwischen 50 und 60 Jahren

E Über 60 Jahre

\section{- 3. Welche Lebenszeitprävalenz wird für die GAS angenommen?}

A $<1 \%$

B $>5 \%$

C $>15 \%$

D $>30 \%$

E $>50 \%$

\author{
- 5. Welche Komorbidität ist führend? \\ A Psychosen \\ B Abhängigkeitserkrankungen \\ C Somatoforme Störungen \\ D Depressive Störungen \\ E Persönlichkeitsstörungen
}

\section{- 6. Die wichtigste Differenzialdiagnose der GAS ist/sind}

A arterieller Hypertonus.

B somatoforme Störungen.

C Persönlichkeitsstörungen.

D Depression.

E Abhängigkeitserkrankungen.

\section{- 7. Wieviel Prozent der GAS-Patienten} werden vermutlich adäquat medikamentös behandelt?
A $10 \%$
B $25 \%$
C $40 \%$
D $50 \%$

E Über $50 \%$

- 8. Welches Medikament bzw. welche Substanz sollte in der Therapie der GAS nicht eingesetzt werden?
A Paroxetin
B S-Citalopram
C Imipramin
A Akut
B Subchronisch
D Intermittierend
E Subakut

D Fluspirilen

E Opipramol

\section{- 9. Welches psychotherapeutische Verfah- ren bei GAS ist durch Therapiestudien am besten untersucht?}

A Gesprächspsychotherapie nach C. Rogers

B Gestalttherapie

C NMDR

D Psychoanalytische Therapie

E Kognitive Verhaltenstherapie

\section{- 10. Mit welcher Therapie-Nonresponse (weniger als 50\% Symptombesserung) müssen Sie bei Einsatz von SSRI und SNR zur Behandlung der GAS rechnen?}

A Bis $10 \%$

B Bis $20 \%$

C Bis $55 \%$

D Bis 70\%

E Über $80 \%$

\section{ANTWORTFORMULAR}

Mit Einreichen dieses Fragebogens erkläre ich mich damit einverstanden, dass die angegebenen Daten zum Zweck der Teilnahmebestätigung gespeichert und bei erfolgreicher Teilnahme an den Einheitlichen Informationsverteiler (EIV) der Ärztekammern weitergegeben werden.

Wichtiger Hinweis:

Eine Auswertung ist künftig nur noch möglich, wenn Sie Ihre EFN auf dem Antwortformular angeben! Nähere Hinweise hierzu unter: www.cme-punkt.de/faq.html
„Krankhafte Sorge“

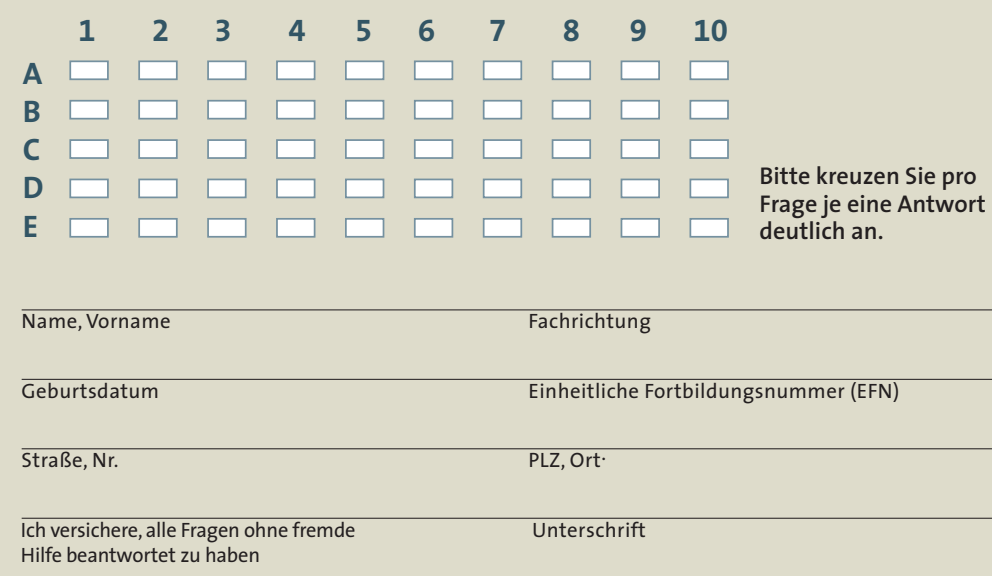

Und so kommen Sie zu Ihren Punkten:

Teilnahme im Internet: unter www.cme-punkt.de. Dort führen wir für Sie ein elektronisches Punktekonto.

Teilnahme per Brief: Fragebogen ausfüllen und mit frankiertem Rückumschlag an: Urban \& Vogel GmbH CME MMW-Fortschr. Med. Postfach 81664 München 